\title{
Applying additive logistic regression to data derived from sensors monitoring behavioral and physiological characteristics of dairy cows to detect lameness
}

\author{
C. Kamphuis, ${ }^{11,2}$ E. Frank, † J. K. Burke, ${ }^{*}$ G. A. Verkerk, ${ }^{*}$ and J. G. Jago* \\ *DairyNZ Ltd., Private Bag 3221, Hamilton 3240, New Zealand \\ †Department of Computer Science, University of Waikato, Private Bag 3105, Hamilton 3240, New Zealand
}

\begin{abstract}
The hypothesis was that sensors currently available on farm that monitor behavioral and physiological characteristics have potential for the detection of lameness in dairy cows. This was tested by applying additive logistic regression to variables derived from sensor data. Data were collected between November 2010 and June 2012 on 5 commercial pasture-based dairy farms. Sensor data from weigh scales (liveweight), pedometers (activity), and milk meters (milking order, unadjusted and adjusted milk yield in the first 2 min of milking, total milk yield, and milking duration) were collected at every milking from 4,904 cows. Lameness events were recorded by farmers who were trained in detecting lameness before the study commenced. A total of 318 lameness events affecting 292 cows were available for statistical analyses. For each lameness event, the lame cow's sensor data for a time period of $14 \mathrm{~d}$ before observation date were randomly matched by farm and date to 10 healthy cows (i.e., cows that were not lame and had no other health event recorded for the matched time period). Sensor data relating to the 14-d time periods were used for developing univariable (using one source of sensor data) and multivariable (using multiple sources of sensor data) models. Model development involved the use of additive logistic regression by applying the LogitBoost algorithm with a regression tree as base learner. The model's output was a probability estimate for lameness, given the sensor data collected during the 14-d time period. Models were validated using leaveone-farm-out cross-validation and, as a result of this validation, each cow in the data set (318 lame and 3,180 nonlame cows) received a probability estimate for lameness. Based on the area under the curve (AUC), results indicated that univariable models had low predictive potential, with the highest AUC values found for liveweight $(\mathrm{AUC}=0.66)$, activity $(\mathrm{AUC}=0.60)$,
\end{abstract}

Received May 5, 2013.

Accepted July 16, 2013.

${ }^{1}$ Corresponding author: claudia.kamphuis@wur.nl

${ }^{2}$ Current address: Chair Group Business Economics, Wageningen University, Hollandseweg 1, 6706 KN, Wageningen, the Netherlands. and milking order $(\mathrm{AUC}=0.65)$. Combining these 3 sensors improved AUC to 0.74. Detection performance of this combined model varied between farms but it consistently and significantly outperformed univariable models across farms at a fixed specificity of $80 \%$. Still, detection performance was not high enough to be implemented in practice on large, pasture-based dairy farms. Future research may improve performance by developing variables based on sensor data of liveweight, activity, and milking order, but that better describe changes in sensor data patterns when cows go lame.

Key words: sensor data, data mining, dairy cow, lameness detection

\section{INTRODUCTION}

Lameness has been grouped with mastitis and infertility as the top 3 dairy cow health issues related to economic losses in the dairy industry (Juarez et al., 2003). Lameness affects welfare negatively, as it is associated with pain (Whay et al., 1997; Bicalho et al., 2007), and decreases farm profitability due to poorer reproductive performance, loss of milk production, and increased costs due to treatment and culling (Tranter and Morris, 1991; Sprecher et al., 1997; Green et al., 2002). Lame cows are usually detected by visual observation of gait and back posture (Sprecher et al., 1997); however, in larger herds, along with the number of cows managed per farm labor unit, visual detection of lame cows becomes more challenging.

Previous studies reported that lameness affects the cow's normal behavior and physiology: lame cows are less active (Juarez et al., 2003; Walker et al., 2008), enter the milking parlor later (Walker et al., 2008), produce less milk (Green et al., 2002), and lose body condition (Walker et al., 2008). Sensing technologies are available that can monitor these behavioral and physiological characteristics of cows on a daily basis. For example, with milk meters and weigh scales, a cow's milk production and liveweight can be regularly monitored. Kamphuis et al. (2013) demonstrated that cows becoming clinically lame have sensor data trends that are significantly different for liveweight, activity, 
milking order, milk yield (produced in the first $2 \mathrm{~min}$ after teat cup attachment and total milk yield), and milking duration compared with cows that do not become clinically lame. Although considerable variation existed in sensor data values between and within lame and nonlame cows, results indicated that sensor data are potentially useful in the detection of lameness.

Data sets containing sensor data are often noisy, due to sensor drift or malfunctioning, and incomplete due to missing values. Additionally, data sets are often imbalanced, as the incidence of lameness is low (Tranter and Morris, 1991; Gibbs, 2010). To analyze this noisy, incomplete, and imbalanced data, it is essential that the modeling technique used can process data with these anomalies and can model nonlinear relationships. Examples of these more sophisticated models in the field of lameness detection are neural networks applied by Pastell and Kujala (2007) and principal components analyses used by Miekley et al. (2013). A model that has not been used in automated detection of lameness is a data-mining technique called decision-tree induction, a commonly used technique for classification problems (Quinlan, 1986), in combination with a boosting process that is known to improve accuracy of classification models (Freund and Schapire, 1996). Decision-tree induction with boosting has proven useful to analyze data with similar anomalies in automated clinical mastitis detection (Kamphuis et al., 2010) where it was able to improve detection performance to a level suggested to be of practical relevance, being $>80 \%$ sensitivity and $>99 \%$ specificity (Hogeveen et al., 2010). These values mean that a model should find at least $80 \%$ of the cows that do have clinical mastitis and at the same time indicate less than $1 \%$ of the healthy cows erroneously. It is unknown what performance targets should be set for a lameness detection model.

The hypothesis for the current study was that sensors currently available on farm to monitor behavioral and physiological characteristics of dairy cows can be used for the detection of lameness. This was tested by applying a boosting technique based on additive logistic regression (Friedman et al., 2000) in combination with a specific type of decision-tree algorithm (regression tree) to variables derived from one sensor (univariable models) and multiple sensors (multivariable models) and assessing their detection performance using leaveone-farm-out cross-validation.

\section{MATERIALS AND METHODS}

Ethics approval was obtained through the Ruakura Animal Ethics Committee (Ruakura, Hamilton, New Zealand; application number 12210) before commencement of the study.
Data were collected from 5 pasture-based dairy farms in the Waikato region of New Zealand between November 2010 and June 2012 (Table 1). All farms except one applied a seasonal spring-calving regimen; one farm had cows calving in spring and autumn. On all farms, cows were milked on a rotary milking platform (Waikato Milking Systems NZ Ltd., Hamilton, New Zealand) fitted with automatic weigh scales and electronic milk meters. All cows had a pedometer (Afikim, Kibbutz Afikim, Israel) fitted to one hind leg for measuring cow activity. The pedometers also contained an electronic cow identification unit. Individual cow and sensor data from each milking session were automatically recorded on herd management software (Frontier; Afikim), with data files generated daily and transferred via the internet to a central database at DairyNZ Ltd. (Hamilton, New Zealand). Cow data included cow identification number and DIM. Sensor data at the cow level included (1) liveweight, (2) activity as the average number of steps per hour between milking sessions, (3) milking order, (4) milk yield in the first 2 min after teat cup attachment, (5) total milk yield, and (6) milking duration. Participating farmers were trained (Healthy Hoof Programme; DairyNZ Ltd.) by accredited veterinarians in detecting and diagnosing lame cows before the study commenced. When a cow was identified as lame, farmers recorded the cow identification number, date of observation, affected limb, and severity of lameness using a 5-point lameness-scoring system (adapted from Sprecher et al., 1997), with scoring categories being (1) normal, (2) mildly lame, (3) moderately lame, (4) lame, and (5) severely lame. Farmers were visited monthly to collect farmer-recorded data on lameness and during these visits lameness-scoring forms were discussed to ensure standardized recording throughout the study period. At the end of the study, data on other health events (e.g., clinical mastitis events and data on AI or natural breeding events) that occurred during the collection period were extracted from the herd management software.

\section{Data Preparation}

Cow and sensor data were automatically recorded in 2 separate data sets. The first data set included information on date, cow identification number, DIM, and data on liveweight and activity measured at both morning and afternoon milkings for each cow for each DIM. The second data set included date, cow identification, and data on milking order, milk yield in the first $2 \mathrm{~min}$ after teat cup attachment, total milk yield, and milking duration. These were also measured during morning and afternoon milking for each cow for each DIM. Milking order was made proportional to the number 
Table 1. Details of data collected from the 5 pasture-based Waikato (New Zealand) dairy farms, including the start date, number of unique cows milked, number of cow milkings, total number of lameness recordings with sensor data, and number of lameness events included in the statistical analyses after excluding the first 30 DIM, lame cows that had other simultaneous health events, and lame cows with less than $10 \mathrm{~d}$ of sensor data before lameness was observed

\begin{tabular}{llcccc}
\hline Item & Start date & $\begin{array}{c}\text { Unique } \\
\text { cows (no.) }\end{array}$ & $\begin{array}{c}\text { Cow milkings } \\
\text { (no.) }\end{array}$ & $\begin{array}{c}\text { Lameness } \\
\text { recordings (no.) }\end{array}$ & $\begin{array}{c}\text { Lameness events } \\
\text { in analyses (no.) }\end{array}$ \\
\hline Farm & & & & & \\
1 & November 27, 2010 & 914 & 261,311 & 164 & 138 \\
2 & December 4, 2010 & 539 & 185,594 & 24 & 52 \\
3 & December 4, 2010 & 814 & 290,956 & 66 & 57 \\
4 & November 30, 2010 & 543 & 146,587 & 73 & 47 \\
5 & December 3, 2010 & 2,094 & 581,142 & 58 & 318 \\
Total & & 4,904 & $1,465,590$ & 385 & \\
\hline
\end{tabular}

of cows milked during that particular milking session and, therefore, values for milking order ranged between 0 and 100. The 2 data sets were merged by date and cow identification number; data on a particular cow and date that were present in just 1 of the 2 data sets were excluded $(2.9 \%$ of the data). Criteria described in Edwards et al. (2013) were used to identify outlier sensor values for milk yield (produced in the first 2 min of teat cup attachment and total milk yield) and milking duration. Sensor values for liveweight and activity were plotted and conservative threshold values were set based on visual judgment of these plots: values for liveweight less than $250 \mathrm{~kg}$ or more than $750 \mathrm{~kg}$ were set as missing ( $<0.5 \%$ of all sensor measurements), as were values for activity less than 25 or more than 1,100 steps per hour $(<0.5 \%$ of all sensor measurements). A seventh variable was created in which the milk yield in the first 2 min after teat cup attachment was made proportional to the total milk yield production for a particular cow during a particular milking session on a particular date. Finally, sensor data measured at morning and afternoon milkings were averaged to get 1 sensor value per day for each variable for each cow in milk. If sensor data were available for 1 milking only, that value was used for that cow for that day.

In total, 466 lameness events were recorded. Lameness events that had no date of observation recorded were excluded $(\mathrm{n}=39)$. Separate lameness events for the same cow for the same affected limb were defined when the time lag between 2 lameness events was $>31$ $\mathrm{d}$; if the period was $\leq 31 \mathrm{~d}$, then the second lameness event recording was excluded $(\mathrm{n}=12)$. Records on other health events were collected, including cow identification number, date of health event, and type of health event (including clinical mastitis, AI, and natural breeding).

Sensor data, information on lameness events, and other health information were merged based on cow identification number and date. Lameness events $(\mathrm{n}=$
30) and other health events without any sensor data were excluded.

\section{Defining Lame and Nonlame Cows}

Lame cows were defined as cows with at least 1 lameness event recorded. To ensure that sensor data used for statistical analyses were not affected by health events other than lameness or by calving events, lameness events were excluded from further analyses when the cow was recorded as having another health event occurring within the interval from $14 \mathrm{~d}$ before $\left(\mathbf{D}_{\text {minus14 }}\right)$ the day of detection $\left(\mathbf{D}_{\mathbf{0}}\right)$ to $7 \mathrm{~d}\left(\mathrm{D}_{\text {plus }}\right)$ after detection (n $=27$ ) and when $\mathrm{D}_{\text {minus14 }}$ fell within the first 30 DIM of that lactation $(\mathrm{n}=34)$.

The $22 \mathrm{~d}$ period (from $\mathrm{D}_{\text {minus14 }}$ to $\mathrm{D}_{\text {plus7 }}$ ) was considered a lameness episode. Lameness episodes with fewer than $10 \mathrm{~d}$ of sensor data from $\mathrm{D}_{\text {minus } 14}$ through $\mathrm{D}_{0}$ were excluded from further analyses $(\mathrm{n}=6)$. Each lameness episode was randomly matched by farm and date with 10 nonlame cows, creating lameness blocks. Nonlame cows were chosen from those cows without a lameness or health event recorded during the matched time period and with at least $10 \mathrm{~d}$ of sensor data from $\mathrm{D}_{\text {minus14 }}$ through $\mathrm{D}_{0}$. Each lameness block, therefore, contained sensor data from 1 lame cow and 10 nonlame cows. The selection procedure allowed lame cows that experienced a lameness event during the study period to contribute sensor data as a nonlame cow during periods they were not lame and nonlame cows to contribute sensor data to more than 1 lameness block.

\section{Model Development and Validation}

From each selected lame and nonlame cow, only sensor data from $D_{\text {minus14 }}$ to $\mathrm{D}_{\text {minus1 }}$ (that is, the $14 \mathrm{~d}$ before the day that the lame cow was detected by the farmer) were included for further analyses, as management intervention might have influenced sensor data from $D_{0}$ 
onwards. Proportional differences in sensor values were calculated between $D_{\text {minusi }}$ and the previous $13 \mathrm{~d}$ for each of the 6 sensor variables and the 1 derived variable. The proportional differences with previous days ( $\mathrm{n}=13$ for each variable) together with the absolute sensor data value on $\mathrm{D}_{\text {minus }}(\mathrm{n}=1$ for each variable) were considered as independent variables to be used for model development.

All the data were used for model development and validation; however, by applying leave-one-farm-out cross-validation, the model was validated using data completely independent from data used for model development. With this cross-validation approach, 5 different models were developed, excluding data from 1 farm in each run; the developed model was then validated using the independent data from the left-out farm. As a result of this cross-validation, each record in the data set $(\mathrm{n}=3,498$, consisting of 318 records from lame and 3,180 records for nonlame cows) was used 4 times for model development and once for validation.

Models were developed using a form of additive logistic regression implemented in an algorithm called LogitBoost (Friedman et al., 2000). LogitBoost uses the boosting process for model development in which a sequence of models or a so-called ensemble model is generated. This ensemble model is built by repeatedly invoking a classification algorithm (the base learner) and by doing so, the ensemble model will fit the training data more closely as the number of boosting iterations grows. The number of boosting iterations used to form the ensemble model is user defined. The boosting process applied by LogitBoost is based on maximizing the conditional likelihood of the ensemble model. The final classification of records is obtained by combining the output of all classification models forming the ensemble model. In the current study, LogitBoost was applied in conjunction with the WEKA REPTree regression tree learner (Hall et al., 2009) as the base learner and the number of iterations was set at 100. A regression tree is a form of decision tree, which is a hierarchical structure consisting of several nodes connected by directed edges (Figure 1). Each node apart from the root node has exactly 1 incoming edge and each node apart from the leaf nodes has at least 2 outgoing edges. These are the internal nodes. At each internal node, an independent variable is tested to decide which edge to follow. In the case of numeric predictors, as in the application used in the current paper, the record's value for the variable that is tested is compared with a numeric threshold stored at the node. If the value is smaller than the threshold, one proceeds along the first edge emanating from the node, otherwise the second one. The REPTree algorithm grows a regression tree from weighted training records by greedily expanding the tree and choos- ing tests at internal nodes and numeric outputs at leaf nodes such that squared error is minimized. To obtain the regression tree's output for a particular record, assessment starts at the root node and traverses along directed edges at the internal nodes until a leaf node containing a numeric value is reached. This numeric value is returned as the tree's output for that record and LogitBoost transforms the output to a probability estimate using

$$
P(\text { lame }=1)=\frac{1}{1+e^{-x}},
$$

where $x$ is the numeric output from the first regression tree in the ensemble model. The larger this numeric output, the closer the probability estimate gets to 1 ; the smaller the value, the closer the probability estimate gets to 0 . To calculate the final probability estimate for lameness in the current study, the LogitBoost algorithm combined the output of the trees from 100 iterations to obtain a probability estimate for lameness using

$$
P(\text { lame }=1)=\frac{1}{1+e^{-\left(x_{1}+x_{2}+\ldots+x_{100}\right)}},
$$

where $x_{1}$ to $x_{100}$ are the numeric output values for a record received from a leaf node for each of the 100 regression trees forming the ensemble model. To avoid overfitting the ensemble model to the training data, which would negatively affect predictive performance on new data, the complexity of the trees generated by REPTree was limited in the current study. More specifically, trees were grown such that no more than 2 edges had to be traversed before reaching a leaf node from the root node. As each edge traversal involved a test on 1 predictor variable, this meant that at most 2 independent variables were inspected before a numeric output was returned from a leaf node (note that it was possible for the same independent variable to be tested at multiple nodes with different numeric thresholds in each node; Figure 1).

Model development and validation were done using independent variables derived from one sensor only (univariable models) and using independent variables derived from a combination of sensors (multivariable models). Models were developed to produce a binary outcome for lameness as well as outcomes for specific lameness scores. In addition, models were developed using all lame and nonlame cows, and using lame cows with a lameness score $\geq 3$ and their matched controls only. It should be noted that the 14 independent variables per sensor are clearly correlated. Correlation may 


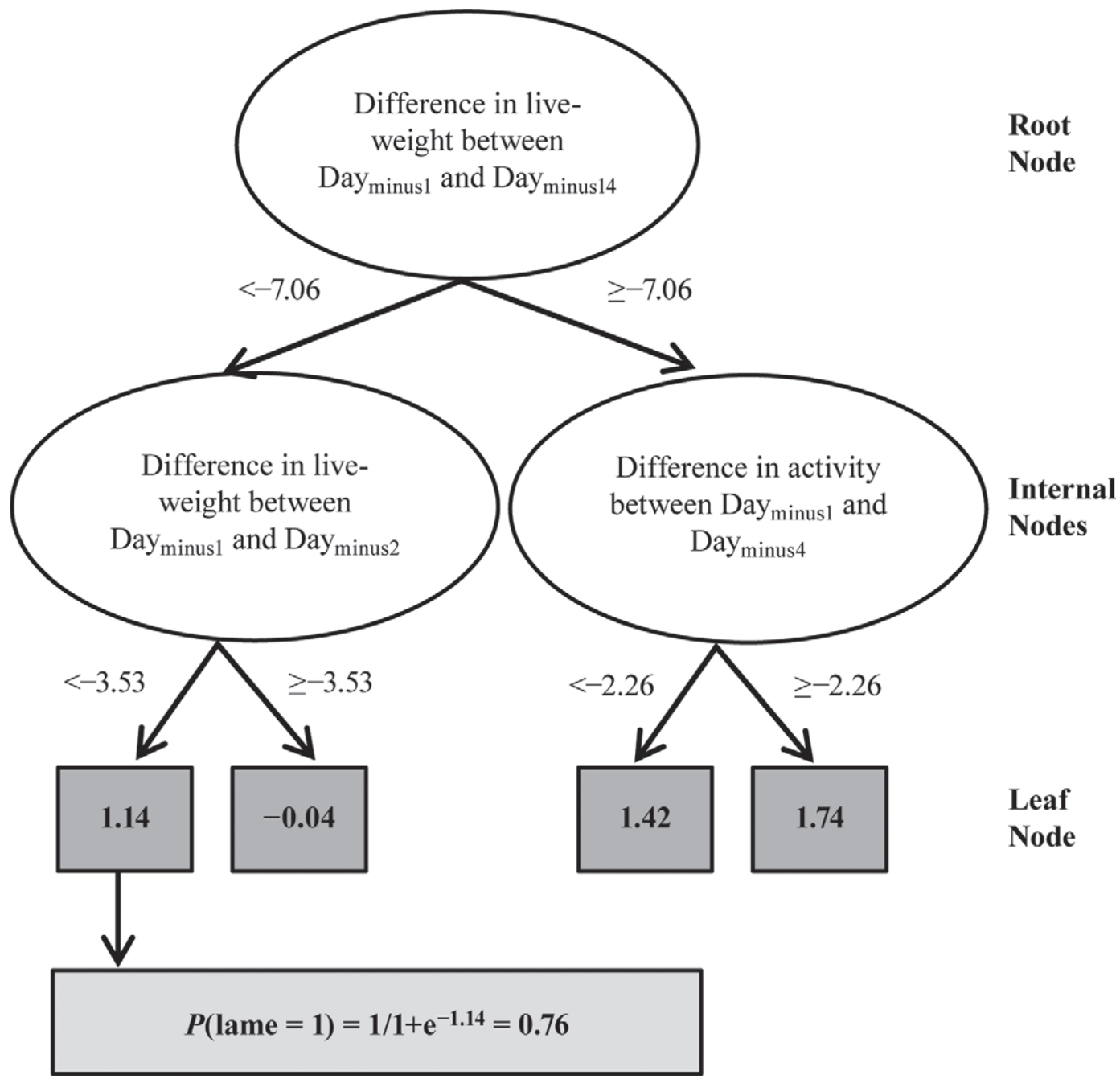

Figure 1. Example of a hypothetical regression tree generated by LogitBoost (Friedman et al., 2000) using a maximum of 2 independent variables to estimate averaged (weighted and rescaled) residuals at the leaf nodes. These residuals are transformed into a probability estimate

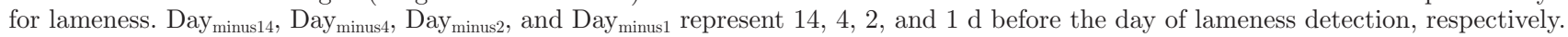

imply that relative prominence of these variables in the model built using LogitBoost does not accurately reflect their true relatively importance. However, in this study, measures of variable importance were not considered; detection performance was solely evaluated when applying the model to independent data.

\section{Performance Measures}

The area under the receiver operating characteristic (ROC) curve was used to evaluate detection performance of different models. This curve is a graphical representation of a model's true positive rate (or sensitivity; in this study, the proportion of lame cows that were correctly classified as lame by the model) against its false positive rate (in this study, the proportion of nonlame cows that were incorrectly classified as lame by the model). When discriminating between lame and nonlame cows, the points on the ROC curve are obtained by changing the threshold value that is used by the model to classify records as lame from the largest possible value to the smallest one (Detilleux et al., 1999; Cortes and Mohri, 2005). In the current study, the additive logistic regression model yielded a probability estimate for lameness for each record in the validation set. These probability estimates were used as possible threshold values to create the ROC curve. When discriminating between lameness scores, a different ROC curve can be drawn for each score by treating that score as the positive category and the union of the other scores as the negative category.

The area under the ROC curve (AUC) summarizes the graphical information into a single quantity. When discriminating between lame and nonlame cows, the 
Table 2. Number of lameness events per farm included in the analysis and distribution of the lameness scores assigned to these events by farmers ${ }^{1}$

\begin{tabular}{|c|c|c|c|c|c|c|}
\hline \multirow[b]{2}{*}{ Item } & \multicolumn{5}{|c|}{ Lameness score } & \multirow[b]{2}{*}{ Total } \\
\hline & 2 & 3 & 4 & 5 & Missing & \\
\hline \multicolumn{7}{|l|}{ Farm } \\
\hline 1 & 38 & 66 & 22 & 4 & 8 & 138 \\
\hline 2 & 8 & 6 & 6 & 1 & 1 & $22(7)$ \\
\hline 3 & 11 & 27 & 10 & 1 & 5 & $54(17)$ \\
\hline 4 & 11 & 24 & 19 & 3 & - & $57(18)$ \\
\hline 5 & 6 & 22 & 18 & - & 1 & $47(15)$ \\
\hline Total & $74(23)$ & $145(46)$ & $75(23)$ & $9(3)$ & $15(5)$ & 318 \\
\hline
\end{tabular}

${ }^{1}$ Lameness scores represent the severity of the recorded lameness event, with score $2=$ mildly lame, score $3=$ moderately lame, score $4=$ lame, and score $5=$ severely lame. Lameness events without a lameness score assigned by the farmer were considered missing. Values in parentheses represent the number of lameness events as a percentage of the total number of lameness events $(n=318)$.

AUC can be interpreted as the probability that the model generates a higher probability estimate for a randomly selected lame cow than for a randomly selected nonlame cow (Hanley and McNeil, 1982). Values for the AUC range between 0.5 and 1 : when a model produces probability estimates at random, it will have an AUC of 0.5 , whereas a perfect ranking (i.e., all lame cows receive a higher probability estimate for lameness than the nonlame cows) will yield an AUC of 1 (Swets, 1988; Cortes and Mohri, 2005).

Two ROC curves may have different shapes within certain threshold value limits but still have the same AUC value (Detilleux et al., 1999). This makes comparison of models' performances using AUC difficult. As a second evaluation measure, the sensitivity of the model was also calculated at 2 predetermined specificity levels (80 and 90\%), where specificity represents the proportion of nonlame cows that were correctly identified as being nonlame by the model. Differences between models in sensitivities at predetermined specificity levels were tested for significance using ANOVA, including farm and model as fixed effects.

Data cleaning, preparation, selection of lame and nonlame cows, and ANOVA were done using SAS software (version 9.2; SAS Institute Inc., Cary, NC). Model building, validation, and retrieving AUC values were done in WEKA (version 3.7.7; University of Waikato, Hamilton, New Zealand; Hall et al., 2009).

\section{RESULTS}

Sensor data were collected from approximately 1.5 million cow milkings from 4,904 dairy cows (Table 1). A total of 385 lameness events had both an observation date and sensor data available; 318 of these were eligible for inclusion in the statistical analyses (Table 1). Most $(43 \%)$ of these eligible lameness events came from farm 1 (Table 2). The biggest proportion (46\%) of the 318 lameness events involved cows that were moderately lame (score 3), $23 \%$ of lameness events involved cows that were mildly lame (score 2), and $26 \%$ of the lameness events involved cows that were lame or severely lame (score 4 or 5 ; Table 2).

When all lame and nonlame cows were included, AUC values ranged between 0.51 and 0.66 for univariable models (Table 3). The univariable models including the variables related to milk production had slightly lower AUC values than the univariable models including liveweight, activity, or milking order (Table 3). Combining sensor data using variables with an AUC $>0.60$ had consistently higher AUC values compared with each univariable model and combining all 3 variables with an AUC $>0.60$ (liveweight, activity, and milking order, and further referred to as the combined model) improved AUC to 0.74 (Table 3). Compared with this combined model, the AUC increased by just 0.04 when all variables were included for model development (Table 3). Using the combined model, AUC values for specific lameness scores were 0.59 for score 2, 0.73 for score 3 and 0.74 for score $\geq 4$. The AUC for the 15 lameness events without a lameness score was 0.52 . Analyses were repeated including only lame cows with a lameness score $\geq 3(\mathrm{n}=229)$ and their matched controls ( $\mathrm{n}=2,290$; Table 3$)$. The general trend was similar for this subgroup: univariable models including variables related to milk production had slightly lower AUC values and combining variables yielded higher AUC values than using univariable variables. The combined model increased the AUC to 0.75 (Table 3) and using this model, the lameness score-specific AUC values were 0.72 for score 3 and 0.71 for score $\geq 4$. The differences between AUC values using all lame cows or only those with lameness score $\geq 3$ were minor.

For the 3 variables with an AUC $>0.60$ and for the combined model, sensitivity levels were calculated at 2 fixed specificity levels using all lame cows and using 


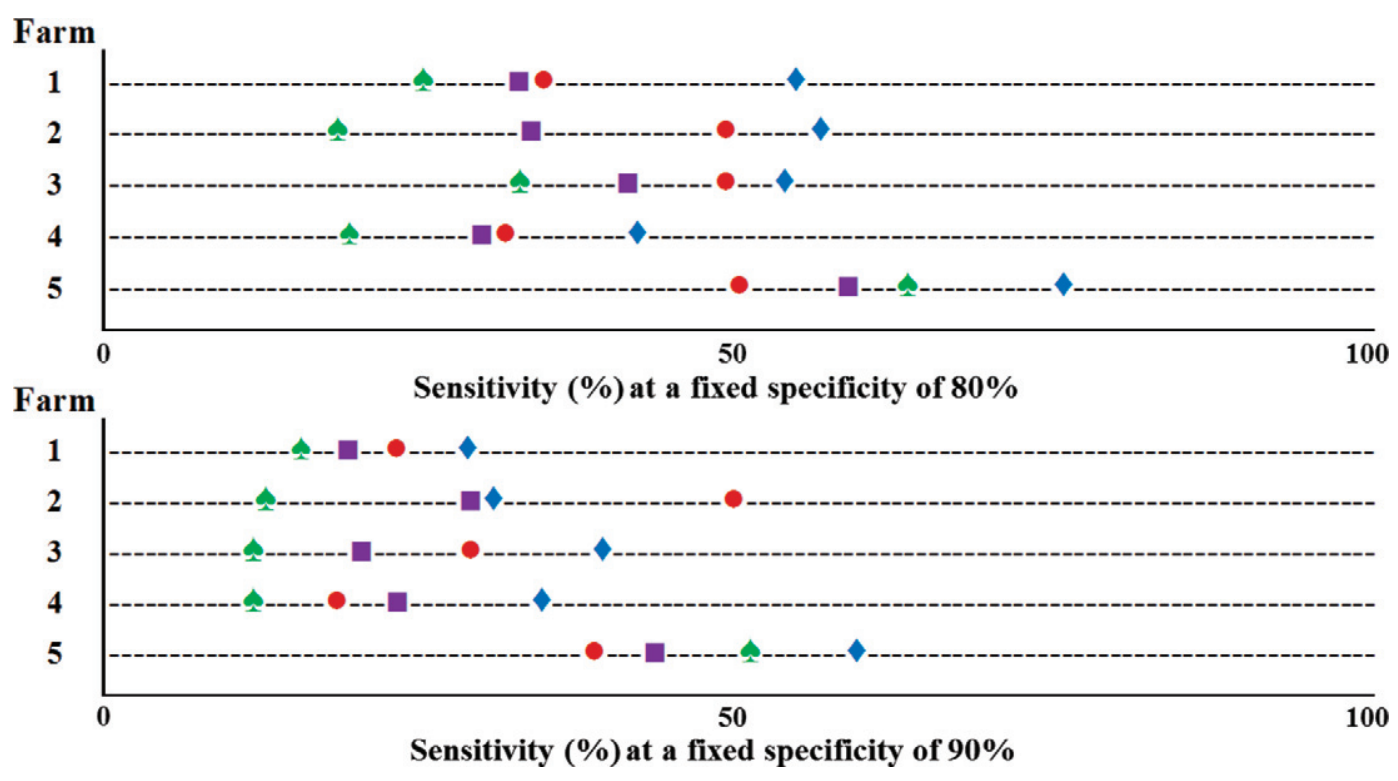

Figure 2. Dot plots of sensitivities at fixed specificities (80 and 90\%) for 3 univariable models (liveweight, $\mathbf{\bullet}$; activity, $\mathbf{p}$; and milking order, ) and a multivariable model combining these 3 variables (combined model, $\$$ ) for each farm. Data from all lame cows $(\mathrm{n}=318)$ were used in these calculations. Color version available in the online PDF.

performance on 4 of the 5 farms. Statistical analyses showed that the difference in performance between the combined model and each univariable model was statistically significant $(P<0.01)$ and that the model only using activity data performed significantly worse than the 3 other models $(P<0.05)$. No significant difference $(P=0.59)$ was observed between the model using liveweight only and the one using milking order only. Results were different at $90 \%$ specificity, where the combined model outperformed the others for 4 of the 5 farms, and where activity performed worst in 4 of the 5 farms. In general, model performance tended to be less consistent at $90 \%$ specificity than at $80 \%$ specificity (Figure 2). At 90\% specificity, the only significant difference $(P<0.01)$ was between the combined model and the model using activity only. When models were developed and validated including lame cows with lameness score $\geq 3$ only, the combined model significantly $(P<0.05)$ outperformed the models using activity and milking order only at $80 \%$ specificity, with no other significant differences between the models. At $90 \%$ specificity and including lame cows with lameness score $\geq 3$, the combined model significantly $(P<0.05)$ outperformed the model using activity only and the model using liveweight only. No other significant differences were observed between models at this specificity level.

\section{DISCUSSION}

Results of this study have confirmed the hypothesis that sensor data available on a growing number of farms are potentially useful for the detection of lameness. Sensor data that gave the best prediction of lameness, based on their AUC value, were liveweight, activity, and milking order. These variables have been associated with lameness in previous studies (e.g., Juarez et al., 2003; Walker et al., 2008). In the current study on pastured cows, the AUC values varied between 0.60 and 0.66 and on their own were not sufficiently high to be of practical use. Combining data from these 3 sensors, however, increased detection performance. The AUC of the combined model was 0.74 when all lame cows were included and 0.75 when only lame cows with a lameness score $\geq 3$ were included (Table 3 ). This result should be interpreted as the combined model having a 74 to $75 \%$ probability of generating a higher-probability estimate for lameness for a randomly selected lame cow than for a randomly selected nonlame cow (Hanley and McNeil, 1982). The slightly better detection performance can be explained by 2 reasons that occur simultaneously when excluding cows with locomotion score 2 and their controls. The first reason is the decreased risk of mislabeling cows with lameness score $\geq 3$ as being healthy and vice versa. The second reason is that differences in sensor data patterns between lame and nonlame cows are more pronounced with increasing lameness score (Kamphuis et al., 2013). Both reasons are linked to the reduction of noise in the data set and this reduction will make it easier for an algorithm to model the data. Adding information based on sensors measuring aspects of milk yield did not improve AUC further. This suggests that sensor data based on milk yield have 
limited potential for lameness detection on the study farms despite previous reports that milk yield is negatively associated with lameness (Green et al., 2002).

The AUC of 0.74 was achieved by combining sensor data from readily available sensors, some of which are already installed on farm but used for different purposes. Sensors that measure activity are usually installed for automated estrus detection (Roelofs et al., 2005; Hockey et al., 2010) and weigh scales can be used to monitor changes in liveweight as a guide for adjusting feeding programs (Alawneh et al., 2011). In the current study, these data were combined for a different purpose (detecting lameness), which would remove the need for farmers to invest in additional (expensive) sensors to automate parts of the farm management processes. The approach used in the current study allowed a large amount of sensor data and a large number of lameness events to be collected in a relatively short period of time. The data set is, therefore, more extensive than in previous reports: 18 cases from 1 farm (Pastell and Kujala, 2007), 58 cases from 1 farm (Bicalho et al., 2007), and 210 cases from 1 farm (Miekley et al., 2013). Furthermore, data collection on multiple farms allowed for leave-one-farm-out cross-validation, thus using completely independent data for model validation. The sensors in the current study were not calibrated as part of the study design and little data cleaning was done to identify erroneous or malfunctioning sensors. This was done deliberately to study if sensors as used on farm could be useful for lameness detection. Even with the noisy data, the combined model outperformed the univariable models significantly at a specificity of $80 \%$ and did this consistently across farms. These results suggest that liveweight, activity, and milking order are likely to be the most useful to identify lame cows, and that lameness can be detected by using information already available on farm better.

The combined model with an AUC of 0.74 used variables based on proportional differences analyzed with a modeling technique called additive logistic regression. These differences, obtained by comparing sensor measurements on different days, are clearly correlated and we cannot preclude that further improvements in detection performance are possible using other techniques. Nevertheless, the AUC of the combined model was higher than the 0.60 reported by Miekley et al. (2013), who applied principal components analysis to develop a lameness detection model that was allowed to assign alerts for up to $3 \mathrm{~d}$ before cows were diagnosed visually as lame. The AUC of the combined model was also higher than the 0.62 reported by Bicalho et al. (2007), who studied a commercially available lamenessdetection system based on force measurements. Bicalho et al. (2007), however, used the cow's reaction to gentle pressure applied by hand to lesions identified at hoof trimming as the gold standard for lameness diagnosis, which is unlikely to be the same as lameness as defined in the current study. Pastell and Kujala (2007) used a probabilistic neural network to detect lame cows using leg-load distribution and reported an AUC of 0.86 . Their model was based on data collected at only 1 farm with limited numbers of cases for model development $(\mathrm{n}=9)$ and validation $(\mathrm{n}=9)$. As a consequence, their model may have overfitted the data used for development. It would be of great interest to test their model using independent data from other farms to confirm the reported performance.

The study farmers were trained to identify lameness but it is still likely that the overall incidence of lameness was underestimated. Previous studies have reported that farmers fail to identify approximately $75 \%$ of lame cows (Whay et al., 2002; Fabian, 2012). Previous work has also reported poor agreement between farmers when assigning locomotion scores (Bicalho et al., 2007). These factors both increase the risk that particularly mildly lame cows (locomotion score 2) are mislabeled as nonlame and hence, visually undetected, but mildly lame cows were likely to have been selected erroneously as nonlame cows. Despite these caveats, the current study demonstrated that sensor data were useful to predict lameness and that AUC values increased with increasing lameness score (from 0.59 for score 2 to 0.74 for score $\geq 4$ ). This result indicates a level of consistency in diagnosing lameness between the enrolled farmers, most likely due to the training that was carried out before the study commenced.

The current study developed prediction models with a binary outcome (lame vs. nonlame), where lame cows were those with a locomotion score $\geq 2$. Whether the applied threshold to define lame and nonlame cows was the most appropriate one is debatable. The current study considered one alternative and that was to exclude mildly lame cows (locomotion score 2) and their controls. This approach may be attractive for performance evaluation purposes; excluding these cows will decrease the noise and with that improve detection performance of a mode. However, from a practical point of view, these mildly lame cows should not be excluded as they are part of real life. This study labeled the mildly lame cows as lame and together with the model having a binary outcome seemed a fair approach: when applied in practice, the model would alert for only a small proportion of these mildly lame cows (lameness score 2 had the lowest AUC). This, however, will likely not be a significant problem, given the mild symptoms and the likelihood of the cow to self-cure without human intervention. When the model would alert for these mildly lame cows, a risk exists that farmers that have 
difficulties with diagnosing mildly lame cows would not recognize the cow as being lame and thus would perceive the alert as false positive. In this situation, the cow could either self-cure or she would deteriorate and the model would pick her up at a later, more severe lameness state. However, for farmers that are capable of diagnosing mildly lame cows, the alerts would be appreciated, as early intervention would be possible. Should future research focus on developing a detection model that predicts specific locomotion scores, mislabeling of cows should be minimized as much as possible.

The AUC obtained in the current study compared with previous reports is a promising result; however, it does not mean that the performance of the combined model is high enough for practical use. When the specificity was fixed at $80 \%$, the combined model had an overall sensitivity of $50 \%$, which increased to $55 \%$ when only lame cows with lameness score $\geq 3$ were included (Table 4). Translating these figures into practice and assuming an incidence rate of 25 cases of lameness per 100 cows per lactation (Gibbs, 2010), a farmer could expect approximately 12 lame cows per month in a 500-cow herd and, by the end of each month, 6 to 7 of these lame cows would be detected correctly. At the same time, however, a specificity of $80 \%$ corresponds to approximately 200 false alerts per 1,000 measurements (Sherlock et al., 2008; i.e., 100 cows would be falsely identified as lame each day in a 500-cow herd). Therefore, although promising, translating the results into practice demonstrates that, based on the current sensor-based variables that represent simple proportional differences, detection performance is not high enough to be implemented on larger pasture-based dairy farms. Future research may improve detection performance by developing variables that better describe the changes in sensor data patterns from liveweight, activity, and milking order when cows go lame.

\section{CONCLUSIONS}

Sensor data that gave the best prediction of lameness, based on their AUC value, were liveweight, activity, and milking order. By combining these 3 sensor data sources, performance increased and this was consistent across farms. Nevertheless, sensor-based variables explored in the current study did not result in a model with a detection performance high enough for practical implementation. To improve detection performance, future research should focus on developing variables based on sensor data of liveweight, activity, and milking order, but that better describe changes in sensor data patterns when cows go lame.

\section{ACKNOWLEDGMENTS}

The authors acknowledge Barbara Dow (DairyNZ Ltd., Newstead, Hamilton, New Zealand) for her statistical support. Also acknowledged are the contributions to the acquisition of the data used in this study by participating farmers. This study was funded by the New Zealand government through the Primary Growth Partnership research program and by New Zealand dairy farmers through DairyNZ Inc.

\section{REFERENCES}

Alawneh, J. I., M. A. Stevenson, N. B. Williamson, N. Lopez-Villalobos, and T. Otley. 2011. Automatic recording of daily walkover liveweight of dairy cattle at pasture in the first 100 days in milk. J. Dairy Sci. 94:4431-4440. http://dx.doi.org/10.3168/jds.20104002 .

Bicalho, R. C., S. H. Cheong, G. Cramer, and C. L. Guard. 2007 Association between a visual and an automated locomotion score in lactating Holstein cows. J. Dairy Sci. 90:3294-3300. http:// dx.doi.org/10.3168/jds.2007-0076.

Cortes, C., and M. Mohri. 2005. Confidence intervals for the area under the ROC curve. Pages 305-312 in Advances in Neural Information Processing Systems 17. L. K. Saul, Y. Weiss, and L. Bottou, ed. MIT Press, Cambridge, MA.

Detilleux, J., J. Arendt, F. Lomba, and P. Leroy. 1999. Methods of estimating areas under receiver operating characteristic curves: Illustration with somatic-cell scores in subclinical intramammary infections. Prev. Vet. Med. 41:75-88. http://dx.doi.org/10.1016/ S0167-5877(99)00054-9.

Edwards, J. P., J. G. Jago, and N. Lopez-Villalobos. 2013. Large rotary dairies achieve high cow throughput but are not more labour efficient than medium sized rotaries. Anim. Prod. Sci. http:// dx.doi.org/10.1071/AN12312.

Fabian, J. 2012. The prevalence of lameness on New Zealand dairy farms: A comparison of farmer perception and mobility scoring. MVS Thesis. Institute of Veterinary, Animal and Biomedical Sciences, Massey University, Palmerston North, New Zealand.

Freund, Y., and R. E. Schapire. 1996. Experiments with a new boosting algorithm. Pages 148-156 in Proc. 13th Intl. Conf. Machine Learning. L. Saitta, ed. Morgan Kaufmann Publishers, San Francisco, CA.

Friedman, J., T. Hastie, and R. Tibshirani. 2000. Additive logistic regression: A statistical view of boosting. Ann. Stat. 28:337-407.

Gibbs, S. J. 2010. Dairy lameness in the South Island. Pages 424-427 in Proc. 4th Australasian Dairy Sci. Symp. Caxton Press, Christchurch, New Zealand.

Green, L. E., V. J. Hedges, Y. H. Schukken, R. W. Blowey, and A. J. Packington. 2002. The impact of clinical lameness on the milk yield of dairy cows. J. Dairy Sci. 85:2250-2256. http://dx.doi. org/10.3168/jds.S0022-0302(02)74304-X.

Hall, M., E. Frank, G. Holmes, B. Pfahringer, P. Reutemann, and I. H. Witten. 2009. The WEKA data mining software: An update. SIGKDD Explor. 11:10-18.

Hanley, J. A., and B. J. McNeil. 1982. The meaning and use of the area under the receiver operating characteristic (ROC) curve. Radiology $143: 29-36$.

Hockey, C. D., J. M. Morton, S. T. Norman, and M. R. McGowan. 2010. Evaluation of a neck mounted 2-hourly activity meter system for detecting cows about to ovulate in two paddock-based Australian dairy herds. Reprod. Domest. Anim. 45:e107-e117. http://dx.doi.org/10.1111/j.1439-0531.2009.01531.x.

Hogeveen, H., C. Kamphuis, W. Steeneveld, and H. Mollenhorst. 2010. Sensors and clinical mastitis - The quest for the perfect alert. Sensors (Basel) 10:7991-8009. http://dx.doi.org/10.3390/s100907991. 
Juarez, S. T., P. H. Robinson, E. J. DePeters, and E. O. Price. 2003. Impact of lameness on behavior and productivity of lactating Holstein cows. Appl. Anim. Behav. Sci. 83:1-14. http://dx.doi. org/10.1016/S0168-1591(03)00107-2.

Kamphuis, C., J. K. Burke, and J. G. Jago. 2013. Cows becoming clinically lame differ in changes in behaviour and physiology compared to cows that do not become clinically lame. Proc. N.Z. Soc. Anim. Prod. 73:5-10.

Kamphuis, C., H. Mollenhorst, J. A. P. Heesterbeek, and H. Hogeveen. 2010. Detection of clinical mastitis with sensor data from automatic milking systems in improved by using decision-tree induction. J. Dairy Sci. 93:3616-3627. http://dx.doi.org/10.3168/jds.20103228 .

Miekley, B., I. Traulsen, and J. Krieter. 2013. Principal component analyses for the early detection of mastitis and lameness in dairy cows. J. Dairy Res. 80:335-343. http://dx.doi.org/10.1017/ S0022029913000290.

Pastell, M. E., and M. Kujala. 2007. A probabilistic neural network model for lameness detection. J. Dairy Sci. 90:2283-2292. http:// dx.doi.org/10.3168/jds.2006-267.

Quinlan, J. R. 1986. Induction of decision trees. Mach. Learn. 1:81106.

Roelofs, J. B., F. J. C. M. van Eerdenburg, N. M. Soede, and B. Kemp. 2005. Pedometer readings for estrous detection and as predictor for time of ovulation in dairy cattle. Theriogenology 64:1690-1703. http://dx.doi.org/10.1016/j.theriogenology.2005.04.004.
Sherlock, R., H. Hogeveen, G. Mein, and M. Rasmussen. 2008. Performance evaluation of systems for automated monitoring of udder health: Analytical issues and guidelines. Pages 275-282 in Proc. Intl. Conf. Mastitis Control: From Science to Practice. T. J. G. M. Lam, ed. Wageningen Academic Publishers, Wageningen, the Netherlands.

Sprecher, D. J., D. E. Hostetler, and J. B. Kaneene. 1997. A lameness scoring system that uses posture and gait to predict dairy cattle reproductive performance. Theriogenology 47:1179-1187. http:// dx.doi.org/10.1016/S0093-691X(97)00098-8.

Swets, J. A. 1988. Measuring the accuracy of diagnostic systems. Science 240:1285-1293.

Tranter, W. P., and R. S. Morris. 1991. A case study of lameness in three dairy herds. N. Z. Vet. J. 39:88-96.

Walker, S. L., R. F. Smith, J. E. Routly, D. N. Jones, M. J. Morris, and H. Dobson. 2008. Lameness, activity time-budgets, and estrus expression in dairy cattle. J. Dairy Sci. 91:4552-4559. http:// dx.doi.org/10.3168/jds.2008-1048.

Whay, H. R., D. C. J. Main, L. E. Green, and A. J. F. Webster. 2002. Farmer perception of lameness prevalence. Pages 355-358 in Proc. 12th Int. Symp. Lameness Ruminants, Orlando, FL. International Veterinary Information Service (IVIS), Ithaca, NY.

Whay, H. R., A. E. Waterman, and A. J. F. Webster. 1997. Associations between locomotion, claw lesions and nociceptive threshold in dairy heifers during the peri-partum period. Vet. J. 154:155-161. 